\title{
Hamiltonian Cayley Digraphs on Direct Products of Dihedral Groups*
}

\author{
Grant Andruchuk, Shonda Gosselin, Yizhe Zeng \\ Department of Mathematics and Statistics, University of Winnipeg, Winnipeg, Canada \\ Email: chuker31@hotmail.com, s.gosselin@uwinnipeg.ca, easyzeng@gmail.com
}

Received May 9, 2012; revised June 10, 2012; accepted June 26, 2012

\begin{abstract}
We prove that a Cayley digraph on the direct product of dihedral groups $D_{2 n} \times D_{2 m}$ with outdegree two is Hamiltonian if and only if it is connected.
\end{abstract}

Keywords: Hamilton Cycle; Cayley Digraph; Dihedral Group

\section{Introduction}

\subsection{Definitions}

For a finite group $G$ and a subset $S$ of $G$, the Cayley digraph $\operatorname{Cay}(G ; S)$ is the directed graph with vertex set $G$ and arcs from $v$ to $v s$ for each $v \in G$ and $s \in S$. The set $S$ is often called the connection set of the digraph $\operatorname{Cay}(G ; S)$, and this digraph is connected if and only if $S$ is a generating set for $G$. The connection set $S$ is said to be minimal if it is a minimal generating set of $G$, and it is said to be minimum if it is a minimal connection set of minimum cardinality. A Hamilton cycle (path) in a digraph of with $n$ vertices is a directed cycle (path) with $n$ vertices. A digraph is said to be Hamiltonian if it has a Hamilton cycle.

Each arc in $\operatorname{Cay}(G ; S)$ of the form $(v, v s)$ is labelled $s$, and called an s-arc. A Hamilton cycle in $\operatorname{Cay}(G ; S)$ can be specified by the sequence of vertices encountered or by the sequence of arcs traversed. In the latter case, it is often more convenient to list the labels of the arcs, rather than the arcs themselves, since for each vertex there is exactly one out-arc with label $S$ for each $s \in S$. An ordered sequence $\left(s_{1}, s_{2}, \cdots, s_{n}\right)$ of the arc labels encountered in a Hamilton cycle is called a Hamiltonian arc sequence. Since Cayley digraphs are vertextransitive, any cyclic shift of a Hamiltonian arc sequence of a Cayley digraph is also a Hamiltonian arc sequence of the digraph, and traversing a Hamiltonian arc sequence of a Cayley digraph starting from any vertex will yield a Hamilton cycle of the digraph. For convenience and brevity, we sometimes omit the commas and brackets from an arc sequence. For an arc sequence $x$, the

${ }^{1}$ The research of S. Gosselin was supported by the University of Winnipeg Board of Regents. symbol $x^{t}$ denotes the concatenation of $t$ copies of $x$. If $v s=w$ for some $v, w \in G$ and $s \in S$, we sometimes write

$$
v \stackrel{s}{\longrightarrow} w
$$

to denote the fact that there is an s-arc from $v$ to $w$ in $\operatorname{Cay}(G ; S)$.

For an integer $n \geq 2$, the symbol $D_{2 n}$ denotes the dihedral group of order $2 n$. For $n \geq 3$ this is the group of symmetries of the regular $n$-gon under the operation of function composition, and it has the presentation $\left\langle R, F \mid R^{n}=e, F^{2}=e, F R F=R^{-1}\right\rangle$, where $R$ is the counterclockwise rotation of $360 / n^{\circ}$ and $F$ is a reflection across any axis of symmetry. For $n=2$ the same presentation can be used to define $D_{4}$. Note that $D_{4} \approx \mathbb{Z}_{2} \times \mathbb{Z}_{2}$.

\subsection{History and Layout of the Paper}

One fundamental problem is that of determining which Cayley digraphs are Hamiltonian. This is a longstanding problem which can be traced back to bell ringing, or campanology, since the orders in which a set of church bells may be rung form a group, and a Hamilton cycle in a Cayley digraph of this group gives a sequence of these bell ringing orders which is pleasing to the ear. The problem is longstanding mainly due to its difficulty. There are several good surveys on the problem, including [1-3], which discusses recent progress and current directions in the more general related problem of finding Hamilton cycles and paths in vertex-transitive graphs.

One of the first elegant results on the problem of the Hamiltonicity of Cayley digraphs is due to Rankin [4], who determined which connected Cayley digraphs on a group $G$ with connection set $\{a, b\}$ are Hamiltonian, in the case where $\left\langle a b^{-1}\right\rangle$ is a normal subgroup of $G$. 
This solves the problem for Cayley digraphs with two generators for a class of groups which includes the Abelian groups, and some Cayley digraphs on solvable groups with two generators. In Section 2 we prove the following theorem.

Theorem 1.1. A Cayley digraph on $D_{2 n} \times D_{2 m}$ with outdegree two is Hamiltonian if and only if it is connected.

This is a new result since such digraphs do not satisfy the hypothesis of Rankin's result. We first prove that if $D_{2 n} \times D_{2 m}$ is generated by two elements then both $n$ and $m$ are odd, and the proof makes use of the following result due to Gaschütz in 1955 [5].

Proposition 1.2. (Gaschütz [5]) Let $G_{1}$ and $G_{2}$ be groups. If $G=G_{1} \times G_{2}$ is finite, then $G$ is generated by two elements if and only if each of the groups $G_{1}, G_{2}, G_{1} / R\left(G_{1}\right) \times G_{2} / R\left(G_{2}\right)$ is generated by two elements, where $R\left(G_{i}\right)$ is the intersection of the maximal proper normal subgroups of $G_{i}$ for $i=1,2$.

\section{Direct Products of Dihedral Groups}

In this section we prove Theorem 1.1. We make use of the following lemma.

Lemma 2.1. If $D_{2 n} \times D_{2 m}$ is generated by two elements, then both $n$ and $m$ are odd.

Proof: Since any dihedral group is generated by two elements, Proposition 1.2 implies that $D_{2 n} \times D_{2 m}$ is generated by two elements if and only if $D_{2 n} / R\left(D_{2 n}\right) \times D_{2 m} / R\left(D_{2 m}\right)$ is generated by two elements, where $R\left(D_{2 n}\right)$ and $R\left(D_{2 m}\right)$ denote the intersections of the maximal normal subgroups of $D_{2 n}$ and $D_{2 m}$, respectively. If $n$ is even and $n \neq 2$, then the normal subgroups of

$$
D_{2 n}=\left\langle R, F \mid R^{n}=e, F^{2}=e, F R F=R^{-1}\right\rangle
$$

are

$$
\left.\left\langle R^{k}\right\rangle \text { (for } 0 \leq k \leq n-1\right),\left\langle R^{2}, F\right\rangle,\left\langle R^{2}, R F\right\rangle \text {. }
$$

Thus the intersection of the maximal normal subgroups of $D_{2 n}$ is $R\left(D_{2 n}\right)=\left\langle R^{2}\right\rangle$. On the other hand, if $n$ is odd, then the normal subgroups of $D_{2 n}$ are all of the form $\left\langle R^{k}\right\rangle$ for $1 \leq k \leq n-1$, and so in this case there is only one maximal normal subgroup, namely $R\left(D_{2 n}\right)=\langle R\rangle$. Note that $D_{2 n} /\left\langle R^{2}\right\rangle \approx D_{2(n-1)}$ and $D_{2 n} /\langle R\rangle \approx \mathbb{Z}_{2}$. Thus Proposition 1.2 implies that if $n$ and $m$ are both even then $D_{2 n} \times D_{2 m}$ is generated by two elements if and only if $D_{2(n-1)} \times D_{2(m-1)}$ is generated by two elements, and if exactly one of $n$ or $m$ is even, say $n$ is even, then $D_{2 n} \times D_{2 m}$ is generated by two elements if and only if $D_{2(n-1)} \times \mathbb{Z}_{2}$ is generated by two elements. Using induction and the fact that $D_{4} \approx \mathbb{Z}_{2} \times \mathbb{Z}_{2}$, we conclude that if $n$ or $m$ is even and $D_{2 n} \times D_{2 m}$ is generated by two elements, then either

$\mathbb{Z}_{2} \times \mathbb{Z}_{2} \times \mathbb{Z}_{2} \times \mathbb{Z}_{2}$ or $\mathbb{Z}_{2} \times \mathbb{Z}_{2} \times \mathbb{Z}_{2}$ is generated by two elements, a contradiction. Hence both $n$ and $m$ must be odd.

Proof of Theorem 1.1: If a Cayley digraph is Hamiltonian, then it is certainly connected. Conversely, if a Cayley digraph on $D_{2 n} \times D_{2 m}$ of outdegree two is connected, then $D_{2 n} \times D_{2 m}$ is generated by two elements. Let $S=\left\{\left(A_{1}, A_{2}\right),\left(B_{1}, B_{2}\right)\right\}$ be a generating set for $D_{2 n} \times D_{2 m}$. By Lemma 2.1, both $m$ and $n$ must be odd. If $A_{i}$ and $B_{i}$ are both rotations in $D_{2 n}$ for some $i \in\{1,2\}$, then $S$ does not generate $D_{2 n} \times D_{2 m}$, a contradiction. Hence at least one of $A_{i}$ and $B_{i}$ is a reflection for $i=1,2$. If $A_{1}, B_{1}, A_{2}, B_{2}$ are all reflections, then every element in $\langle a, b\rangle$ is an ordered pair of reflections or an ordered pair of rotations, a contradiction. If a rotation in $\left\{A_{1}, B_{1}\right\}$ does not generate the cyclic subgroup of rotations of $D_{2 n}$, or if a rotation in $\left\{A_{2}, B_{2}\right\}$ does not generate the cyclic subgroup of rotations of $D_{2 m}$, then $S$ does not generate all of $D_{2 n} \times D_{2 m}$, a contradiction. Thus any 2-element generating set $S$ must have one of the following two forms:

1) $S=\left\{a=\left(R_{n}, F_{m}\right), b=\left(F_{n}, R_{m}\right)\right\}$ for reflections $F_{n} \in D_{2 n}$ and $F_{m} \in D_{2 m}$, and rotations $R_{n} \in D_{2 n}$ and $R_{m} \in D_{2 m}$ or orders $n$ and $m$, respectively.

We will show that

$$
\left(\left(a^{n-1} b\right)^{2 m-1} a^{n}\right)^{2}
$$

is a Hamiltonian arc sequence in $\operatorname{Cay}\left(D_{2 n} \times D_{2 m} ;\{a, b\}\right)$. Each element of $D_{2 n} \times D_{2 m}$ may be written uniquely in the form $\left(R_{n}^{i} F_{n}^{j}, R_{m}^{k} F_{m}^{\ell}\right)$ where $0 \leq i<n, 0 \leq k<m$, and $0 \leq j, \ell<2$. For convenience, we will represent the element $\left(R_{n}^{i} F_{n}^{j}, R_{m}^{k} F_{m}^{\ell}\right)$ by the ordered string ijk $\ell$. Following the arc labelled $a$ from a given vertex ijk $\ell$ of the digraph increases the value of $i$ by 1 modulo $n$ if $j=0$, decreases the value of $i$ by 1 modulo $n$ if $j=1$, fixes the value of $j$, increases the value of $\ell$ by 1 modulo 2, and fixes the value of $k$. Similarly, following the arc labelled $b$ from a given vertex ijk $\ell$ of the digraph increases the value of $k$ by 1 modulo $m$ if $\ell=0$, decreases the value of $k$ by 1 modulo $m$ if $\ell=1$, fixes the value of $\ell$, increases the value of $j$ by 1 modulo 2, and fixes the value of $i$.

Starting for the identity vertex 0000 and following the sequence $a^{n-1}$, we form a path which visits each vertex of the form $i 00 \ell$, for which $i$ and $\ell$ have the same parity, exactly once. Now following $b a^{n-1}$ we extend this path to visit each vertex of the form i11 $\ell$ where $i \equiv \ell \bmod 2$. Again following $b a^{n-1}$, we visit each vertex of the form $i 02 \ell$ where $i \equiv \ell \bmod 2$. Continuing in this way, starting from 0000 and following the arc sequence $a^{n-1}\left(b a^{n-1}\right)^{m-1}$, we form a path which visits each vertex 
of the form $i j k \ell$ where both $i \equiv \ell \bmod 2$ and $j \equiv k \bmod 2$. Starting from the last vertex on this path and following the arc sequence $\left(b a^{n-1}\right)^{m}$, we visit each vertex of the form $i j k \ell$ where $i \equiv \ell \bmod 2$ and $j \neq \equiv k \bmod 2$. In total, starting from the identity vertex 0000 and following arc sequence $\left(a^{n-1} b\right)^{2 m-1} a^{n-1}$, we form a path which visits each vertex of the form ijk $\ell$ exactly once, where $i \equiv \ell \bmod 2$. Now following arc $a$ from the last vertex on this path, we land on the first vertex $(n-1) 1(m-1) 1$ of our path of the form ijk $\ell$ with $i \neq \equiv \ell \bmod 2$. Now repeating the arc sequence $\left(a^{n-1} b\right)^{2 m-1} a^{n-1}$, we visit each vertex of the form ijk $\ell$ with $i \neq \equiv \bmod 2$ exactly once, and finish on the vertex $(n-1) 001$. Thus we have formed a Hamilton path. Finally, following arc $a$, we land back on the identity vertex 0000 . Hence the complete arc sequence

$$
\left(a^{n-1} b\right)^{2 m-1} a^{n-1} a\left(a^{n-1} b\right)^{2 m-1} a^{n-1} a=\left(\left(a^{n-1} b\right)^{2 m-1} a^{n}\right)^{2} \text { is a }
$$

Hamiltonian arc sequence. This arc sequence is shown in

Figure 1 for the case where $n=5$ and $m=3$.

2) $S=\left\{a=\left(F_{n}, R_{m}\right), b=\left(F_{n} R_{n}, F_{m}\right)\right\}$ for reflections $F_{n} \in D_{2 n}$ and $F_{m} \in D_{2 m}$, and rotations $R_{n} \in D_{2 n}$ and $R_{m} \in D_{2 m}$ or orders $n$ and $m$, respectively.

In this case, we show that

$$
\left(a^{2 m-1} b\right)^{2 n}
$$

is a Hamiltonian arc sequence in $\operatorname{Cay}\left(D_{2 n} \times D_{2 m} ;\{a, b\}\right)$. First we will prove that this arc sequence traces out a walk in $\operatorname{Cay}\left(D_{2 n} \times D_{2 m} ;\{a, b\}\right)$ which visits all vertices, then we will show that this walk is closed. Note that each vertex of this graph can be written uniquely in the form $\left(F_{n}^{i} R_{n}^{j}, F_{m}^{k} R_{m}^{\ell}\right)$ where $i, k \in \mathbb{Z}_{2}, \quad j \in \mathbb{Z}_{n}$ and $\ell \in \mathbb{Z}_{m}$.

To see that the arc sequence $\left(a^{2 m-1} b\right)^{2 n}$ visits all vertices of the digraph, notice that starting at any vertex $v=\left(F_{n}^{i} R_{n}^{j}, F_{m}^{k} R_{m}^{\ell}\right)$ and following arc sequence $a^{2 m-1}$, we visit all vertices in the coset of $\langle a\rangle$ which contains $v$. Hence, starting from $v$, if the vertex $x$ reached by arc sequence $\left(a^{2 m-1} b\right)^{s}$ and the vertex $y$ reached by arc sequence $\left(a^{2 m-1} b\right)^{t}$ lie in different cosets of $\langle a\rangle$ whenever $0 \leq s<t \leq 2 n-1$, we can conclude that the arc sequence $\left(a^{2 m-1} b\right)^{2 n}$ traces out a walk which visits all vertices of the digraph. Suppose, for the sake of contradiction, that $x$ and $y$ lie in the same coset of $\langle a\rangle$. We have

$$
\begin{aligned}
- & \left(F_{n}^{i} R_{n}^{j}, F_{m}^{k} R_{m}^{\ell}\right) \stackrel{a}{\longrightarrow} \\
& \left(F_{n}^{(i+1) \bmod 2} R_{n}^{(-j) \bmod n}, F_{m}^{k} R_{m}^{(\ell+1) \bmod m}\right),
\end{aligned}
$$

- $\left(F_{n}^{i} R_{n}^{j}, F_{m}^{k} R_{m}^{\ell}\right) \stackrel{b}{\longrightarrow}$ $\left(F_{n}^{(i+1) \bmod 2} R_{n}^{(-j+1) \bmod n}, F_{m}^{(k+1) \bmod 2} R_{m}^{-\ell \bmod m}\right)$,

- $\left(F_{n}^{i} R_{n}^{j}, F_{m}^{k} R_{m}^{\ell}\right) \stackrel{a^{2 m-1} b}{\longrightarrow}$ $\left(F_{n}^{i} R_{n}^{(j+1) \bmod n}, F_{m}^{(k+1) \bmod 2} R_{m}^{(-\ell+1) \bmod m}\right)$.

It is easy to see that traveling by a sequence of $a$-arcs doesn't change the exponent of $F_{m}$. Also, each time a vertex travels by an $a$-arc, its first coordinate alternates between $F_{n}^{(i+1) \bmod 2} R_{n}^{(-j) \bmod n}$ and $F_{n}^{i} R_{n}^{j}$, and each time a vertex travels by the arc sequence $a^{2 m-1} b$, its second coordinate alternates between $F_{m}^{(k+1) \bmod 2} R_{m}^{(-\ell+1) \bmod m}$ and $F_{m}^{k} R_{m}^{\ell}$. Hence

$$
\begin{aligned}
& \left(F_{n}^{i} R_{n}^{j}, F_{m}^{k} R_{m}^{\ell}\right) \stackrel{\left(a^{2 m-1} b\right)^{s}}{\longrightarrow} \\
& \left\{\begin{array}{cc}
\left(F_{n}^{i} R_{n}^{(j+s) \bmod n}, F_{m}^{(k+s) \bmod 2} R_{m}^{(-\ell+1) \bmod m}\right), & s \text { odd } \\
\left(F_{n}^{i} R_{n}^{(j+s) \bmod n}, F_{m}^{(k+s) \bmod 2} R_{m}^{\ell}\right), & s \text { even }
\end{array}\right.
\end{aligned}
$$

and

$$
\begin{aligned}
& \left(F_{n}^{i} R_{n}^{j}, F_{m}^{k} R_{m}^{\ell}\right) \stackrel{\left(a^{2 m-1} b\right)^{t}}{\longrightarrow} \\
& \left\{\begin{array}{cc}
\left(F_{n}^{i} R_{n}^{(j+t) \bmod n}, F_{m}^{(k+t) \bmod 2} R_{m}^{(-\ell+1) \bmod m}\right), & t \text { odd } . \\
\left(F_{n}^{i} R_{n}^{(j+t) \bmod n}, F_{m}^{(k+t) \bmod 2} R_{m}^{\ell}\right), & t \text { even }
\end{array}\right.
\end{aligned}
$$

Since $x$ and $y$ are in the same coset of $\langle a\rangle, y$ can be reached from $x$ through a sequence of $a$-arcs, which does not change the exponent of $F_{m}$ in the second coordinate. Thus

$$
k+s \equiv k+t(\bmod 2)
$$

and so

$$
s \equiv t(\bmod 2) .
$$

Also, since the exponent of $F_{n}$ in $x$ and $y$ are equal, $y$ can be reached from $x$ by a sequence of $a-$ arcs of even length. This implies that

$$
j+s \equiv j+t(\bmod n)
$$

and thus

$$
s \equiv t(\bmod n) .
$$

Since $0 \leq s<t \leq 2 n-1$, this implies $t=s+n$, so

$$
t \equiv s+n(\bmod 2)
$$

But then since $n$ is odd we have

$$
t \equiv s+1(\bmod 2),
$$

which contradicts (1). We conclude that $x$ and $y$ lie in different cosets of $\langle a\rangle$, and so the walk traced by the arc sequence $\left(a^{2 m-1} b\right)^{2 n}$ visits every vertex of the digraph.

To show the walk is closed, we choose an initial vertex $v=\left(F_{n}^{i} R_{n}^{j}, F_{m}^{k} R_{m}^{\ell}\right)$ and observe that 


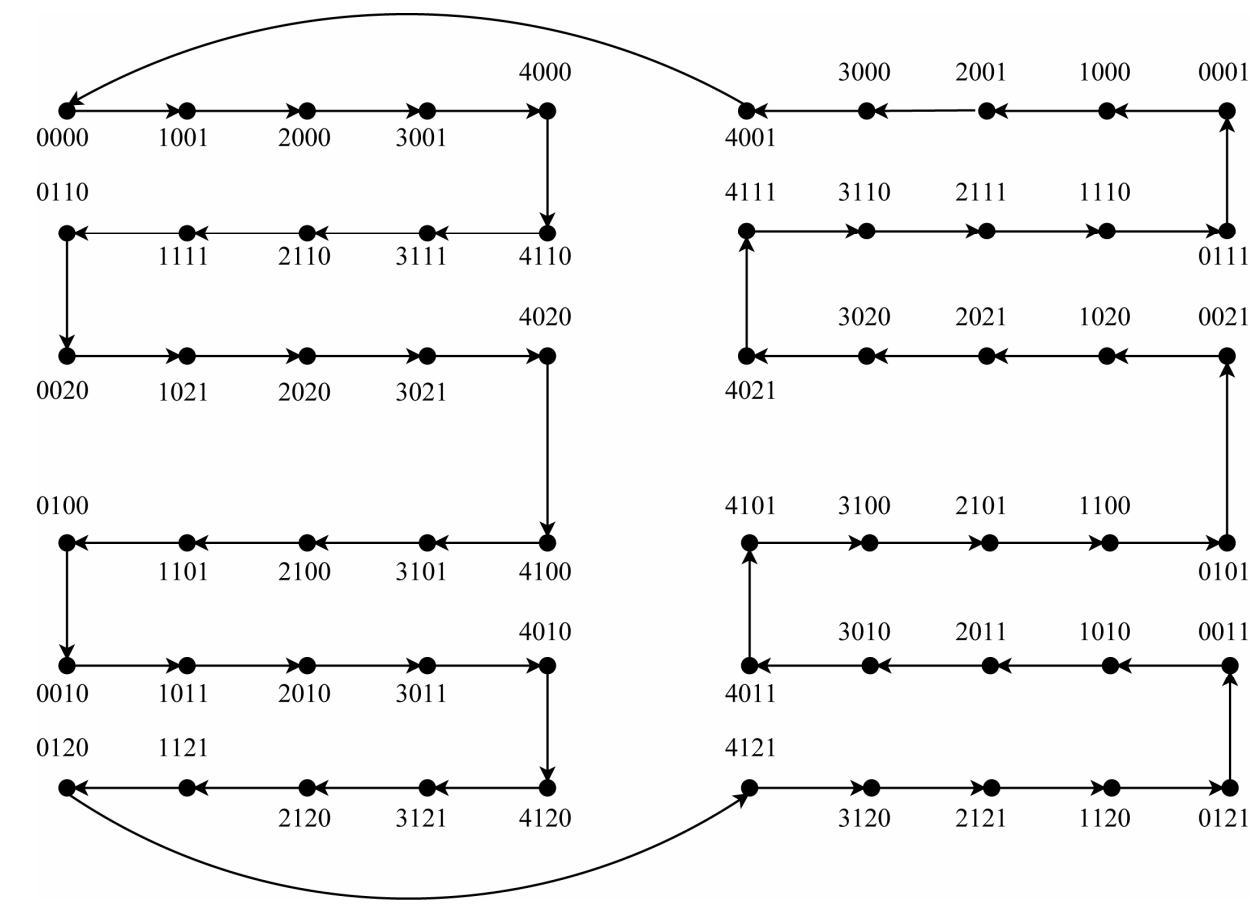

Figure 1. Hamilton cycle in the Cayley digraph on $D_{10} \times D_{6}$ with connection set $\left\{a=\left(R_{5}, F_{3}\right), b=\left(F_{5}, R_{3}\right)\right\}$. The label ijkl denotes the vertex $\left(R_{5}^{i} F_{5}^{j}, R_{3}^{k} F_{3}^{l}\right)$.

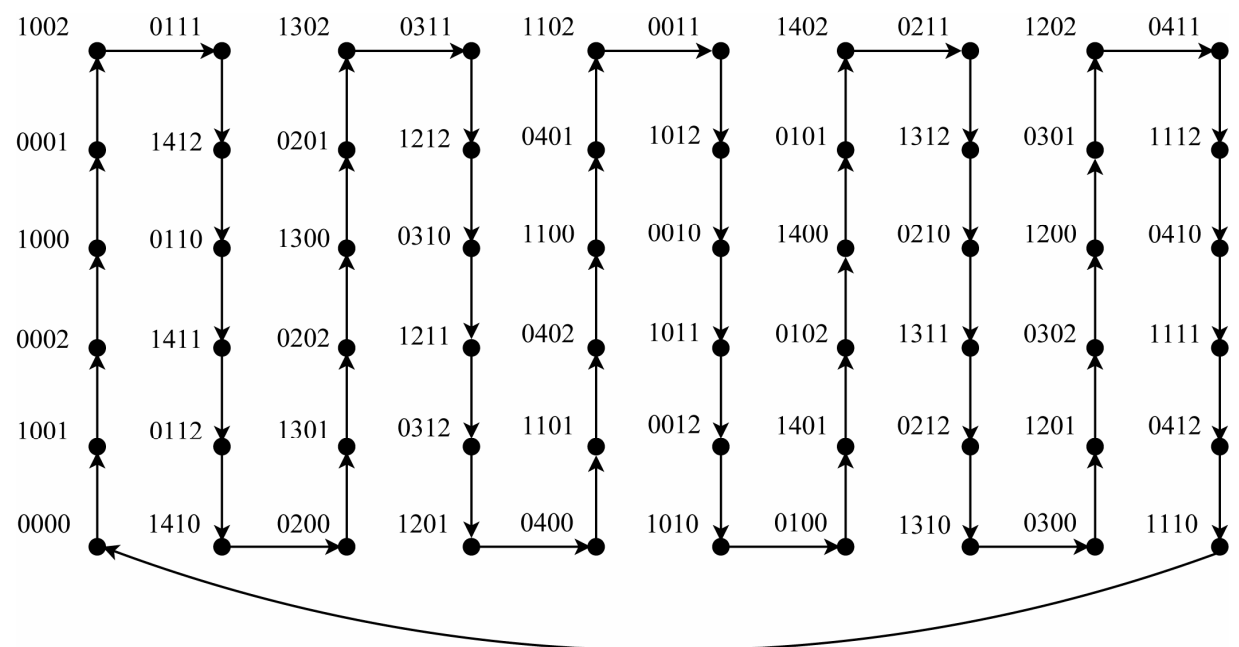

Figure 2. Hamilton cycle in the Cayley digraph on $D_{10} \times D_{6}$ with connection set $\left\{a=\left(F_{5}, R_{3}\right), b=\left(F_{5} R_{5}, F_{3}\right)\right\}$. The label ijkl denotes the vertex $\left(F_{5}^{i} R_{5}^{j}, F_{3}{ }^{k} R_{3}^{l}\right)$.

$$
\left(F_{n}^{i} R_{n}^{j}, F_{m}^{k} R_{m}^{\ell}\right) \stackrel{\left(a^{2 m-1} b\right)^{2 n}}{\longrightarrow}\left(F_{n}^{i} R_{n}^{(j+2 n) \bmod n}, F_{m}^{(k+2 n) \bmod 2} R_{m}^{\ell}\right)
$$

which reduces to the initial vertex $v$.

Finally, since the arc sequence $\left(a^{2 m-1} b\right)^{2 n}$ traces out a walk of length $4 m n=\left|D_{2 n} \times D_{2 m}\right|$ which is closed and visits every vertex of the digraph, we conclude that it is a Hamiltonian arc sequence. This arc sequence is shown in Figure 2 for the case where $n=5$ and $m=3$.

\section{REFERENCES}

[1] S. Curran and J. Gallian, "Hamiltonian Cycles and Paths in Cayley Graphs and Digraphs-A Survey," Discrete Mathematics, Vol. 156, No. 1-3, 1996, pp. 1-18. doi:10.1016/0012-365X(95)00072-5

[2] J. Gallian and D. Witte, "A Survey: Hamiltonian Cyles in Cayley Graphs," Discrete Mathematics, Vol. 51, No. 3, 1984, pp. 293-304. doi:10.1016/0012-365X(84)90010-4

[3] K. Kutnar and D. Marušič, "Hamilton Cycles and Paths in 
Vertex-Transitive Graphs-Current Directions," Dicrete Mathematics, Vol. 309, No. 17, 2009, pp. 5491-5500. doi:10.1016/j.disc.2009.02.017

[4] R. A. Rankin, "A Campanological Problem in Group Theory," Proceedings of the Cambridge Philosophical Society, Vol. 44, No. 1, 1948, pp. 17-25.

\section{doi:10.1017/S030500410002394X}

[5] W. Gaschütz, "Zu Einem von B. H. und H. Neumann Gestellten Problem," Mathematische Nachrichten, Vol. 14, No. 4-6, 1955, pp. 249-252. doi:10.1002/mana.19550140406 